\title{
Enfermedad pulmonar obstructiva crónica: Mirada actual a una enfermedad emergente
}

\author{
RAFAEL SILVA O.
}

Unidad de Enfermedades

Respiratorias y Servicio de Medicina.

Hospital Regional de Talca. Escuela de Medicina Universidad de Talca.

Recibido el 22 de enero de 2010, aceptado el 3 de noviembre de 2010 Correspondencia a: Dr. Rafael Silva O Dos Norte 360, Talca. Fono: 71-413947 Fax: 71-413951 E-mail: rafaelsilvao@gmail.com a enfermedad pulmonar obstructiva crónica (EPOC) se proyecta como la tercera causa $\checkmark$ de muerte en el mundo en el año $2020^{1}$. La prevalencia y el impacto de esta enfermedad sobre la población se espera aumente por el envejecimiento y el alza en las tasas de tabaquismo de la población, tanto en países desarrollados como subdesarrollados. La prevalencia mundial en la población general se estima en alrededor de $1 \%{ }^{2}$ y en mayores de 40 años en $10 \%{ }^{3}$. En Chile, en la ciudad de Santiago, el estudio Platino ${ }^{4}$ demostró una prevalencia ajustada de $14,5 \%$.

En Estados Unidos de Norteamérica se ha descrito un aumento sostenido de la mortalidad por EPOC entre los años 1970 y $2002^{5}$, lo que ha provocado creciente interés, en todo el mundo y en Chile, por conocer más sobre esta enfermedad, con un aumento sustancial de la investigación en los últimos años, mejorando el conocimiento respecto las características clínicas, fisiopatológicas y, sobre todo, el disponer de nuevas herramientas terapéuticas para los pacientes portadores de EPOC.

\section{Definición}

La iniciativa GOLD (Global Iniciative for Chronic Obstructive Lung Disease) ha definido EPOC como "una enfermedad prevenible y tratable, con afectación sistémica extrapulmonar que puede contribuir a la gravedad en algunos pacientes. El componente pulmonar se caracteriza por una limitación al flujo de aire que no es completamente reversible y la limitación al flujo de aire es por lo general progresiva y se asocia con una respuesta 
inflamatoria pulmonar anómala a partículas o gases nocivos"'.

GOLD clasifica la severidad de los pacientes de acuerdo a la alteración del volumen espirado del primer segundo $\left(\mathrm{VEF}_{1}\right)$ (Figura 1 ) siendo una definición operacional con la ventaja de la simplicidad pero, como lo demuestra una cohorte del estudio ECLIPSE $^{7}$, el VEF no muestra la heterogeneidad de esta enfermedad. Respecto la reversibilidad, Tashkin ${ }^{8}$ mostró modificación de más de $12 \%$ del VEF respecto el basal, con el uso de salbutamol más bromuro de ipatropio, en más de $50 \%$ de los pacientes reclutados en su estudio clínico, con lo que, posiblemente, se está excluyendo a casi la mitad de los pacientes portadores de EPOC en los protocolos de investigación.

\section{Mortalidad por EPOC}

Por años se consideró que los pacientes portadores de EPOC, específicamente aquellos con daño funcional avanzado, eran pacientes terminales a los que se les podía ofrecer pocas alternativas terapéuticas, con un pronóstico ominoso y una alta mortalidad a corto plazo. Esta mirada pesimista ha ido cambiando, entre otras razones, por las nuevas alternativas terapéuticas disponibles y por el mayor conocimiento de su patogénesis.

Por otro lado, no es fácil atribuir mortalidad específica a EPOC, porque es distinto "morir de EPOC" que "morir con EPOC". Se ha encontrado, al reanalizar algunos estudios clínicos, que las discrepancias entre las causas originalmente adjudicadas por los autores y las detectadas por comités independientes en algunos casos pueden llegar al $20 \%{ }^{9}$, por lo que se recomienda tener siempre un comité independiente al grupo investigador ,el que adjudicará la causa de muerte en los protocolos $\mathrm{EPOC}^{10}$. De hecho, el primer protocolo de investigación que utilizó esta metodología fue el estudio TORCH ${ }^{11}$. Este estudio encontró que los pacientes portadores de EPOC fallecen por causa cardiaca en $27 \%$ y por causa respiratoria, $35 \%$. La mortalidad relacionada con EPOC fue 41,3\%.

Respecto la mortalidad por EPOC, el estudio Platino II ${ }^{12}$, con datos presentados en el Congreso Europeo de Enfermedades Respiratorias del año 2009, encontró que en pacientes estadio GOLD II, el odds ratio de mortalidad fue de 4,2. Este dato pone en discusión la intervención terapéutica temprana y agresiva para este grupo de pacientes.

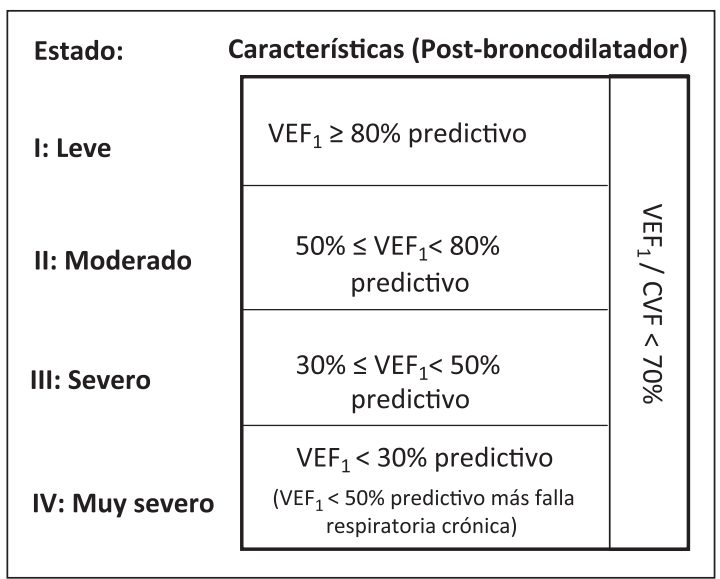

Figura 1. Clasificación EPOC según alteración $V E F_{1} / C V F$ y VEF post-broncodilatador.

\section{Historia natural}

Fletcher y Peto ${ }^{13}$ publicaron en 1977 un artículo clásico, que muestra el riesgo de las personas que fuman y las diferencias entre los que mantienen el habito tabáquico a través de los años, con deterioro progresivo del $\mathrm{VEF}_{1}$, respecto los que abandonan el tabaco (Figura 2). Hoy sabemos que el daño provocado por el cigarrillo en la vía aérea es pre$\mathrm{COZ}$, con alteraciones moleculares, bioquímicas y funcionales antes de que aparezcan síntomas reconocidos por los pacientes ${ }^{14}$. Recientemente se ha demostrado que en los estadios tempranos de la enfermedad, específicamente en los estadios GOLD I, la alteración ventilación/perfusión es desproporcionadamente elevada en relación a la limitación del flujo aéreo, sugiriendo que en EPOC inicialmente se afecta la vía aérea pequeña, el parénquima y los vasos pulmonares, existiendo una mínima alteración espirométrica, por lo que las alteraciones del intercambio gaseoso se desarrollan precozmente ${ }^{15}$.

\section{Actitud nihilista versus mirada optimista}

Hay un sub-diagnóstico de EPOC, posiblemente por el bajo uso de espirometrías en atención primaria ${ }^{16}$, lo que sumado a un diagnóstico erróneo de $64 \%$, detectado en un estudio realizado en cinco ciudades latinoamericanas ${ }^{17}$, más la subpercepción de su enfermedad por parte de estos 


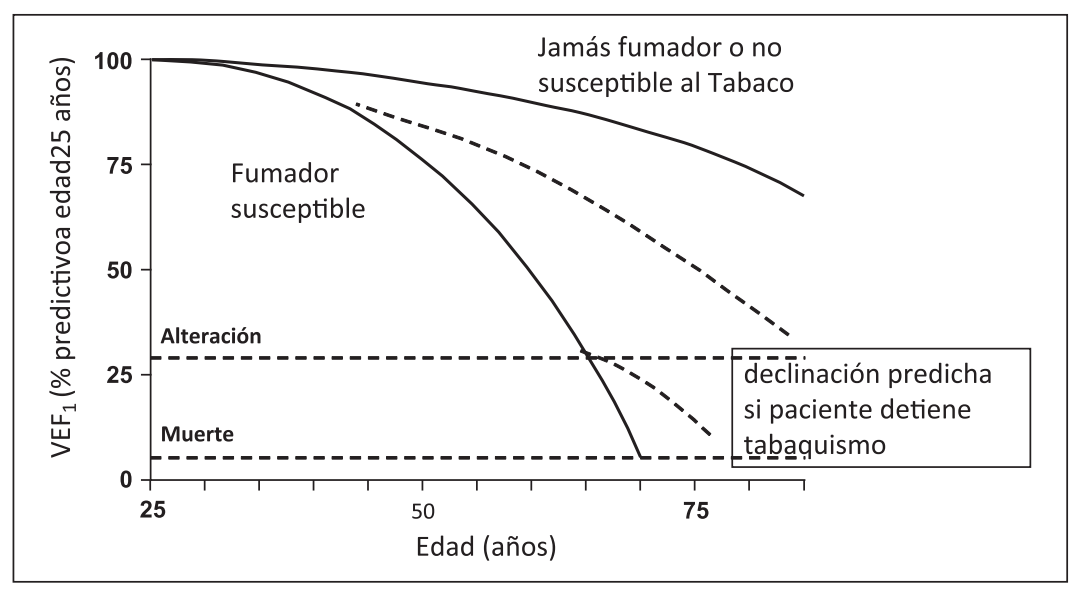

Figura 2. Curva de Fletcher \& Peto: Declinación del $\mathrm{VEF}_{\text {, en }}$ relación a consumo de tabaco en fumadores susceptibles. Adaptado con permiso de referencia 13. pacientes ${ }^{18}$, indica que el desafío es fomentar el conocimiento de la EPOC.

Celli $^{19}$ nos ha enseñado, sobre la base de la evidencia actual, que una mirada nihilista es totalmente injustificada. EPOC no sólo es una enfermedad prevenible sino que también tratable. El enfoque actual es dejar de mirar esta enfermedad como exclusivamente pulmonar, enfocándola como una patología con importantes consecuencias sistémicas. Debemos agregar que hoy conocemos algunas intervenciones terapéuticas, que detallaremos más adelante, que impactan en mortalidad, en calidad de vida, en el número de exacerbaciones y en la capacidad de ejercicio. Por tanto, el futuro de esta enfermedad está marcado por el creciente conocimiento de su patogénesis, por el mayor conocimiento de sus distintos fenotipos y por el advenimiento de nuevas terapias.

\section{Tabaquismo y EPOC}

El habito tabáquico es un factor de riesgo primario para padecer EPOC, pero no todos los fumadores desarrollan la enfermedad. Hay susceptibilidad genética y hay factores exógenos involucrados ${ }^{20}$. En los fumadores susceptibles hay una prevalencia mayor de alteraciones de la función pulmonar y una mayor reducción anual del $\mathrm{VEF}_{1}$. Está demostrado que el humo del cigarrillo activa macrófagos y células epiteliales de la vía aérea desencadenando una cascada en la que participan factores quimiotácticos de los neutrófilos, que finalmente activan proteasas, dando lugar a un desbalance proteasas-antiproteasas, que conduce al daño tisular que se observa en estos pacientes ${ }^{21}$.

\section{Folículos linfoides y autoinmunidad}

Hogg $^{22}$ detectó en pacientes que abandonaron el hábito tabáquico entre 4 y 9 años antes, una vía aérea pequeña con importantes exudados inflamatorios e infiltración de la pared por células inflamatorias inmunes, innatas y adaptativas, formando folículos linfoides. El rol de estos folículos está en estudio. Se ha sugerido que pueden jugar un papel en la respuesta inmune de la vía aérea en el paciente con EPOC y un efecto protector sobre la colonización bacteriana presente en estos pacientes o podrían tener un efecto dañino si participaran en una respuesta autoinmune $e^{23}$. Se ha encontrado que EPOC está asociada con autoanticuerpos contra el epitelio de la vía aérea y contra las células endoteliales pulmonares. Esta es un área potencial de estudios para encontrar posibles terapias inmunomoduladores ${ }^{24}$. Sin embargo, aunque hay evidencia circunstancial indirecta y directa del rol de la autoinmunidad en la EPOC, no se ha establecido una relación causa-efecto y, como ya se comentó, se requieren más investigaciones para aclarar el rol de los folículos linfoides y la autoinmunidad en la patogénesis de $\mathrm{EPOC}^{25}$.

\section{Fenotipos y caracterización clínica}

Se han descrito 2 morfotipos clásicos: A) "Abotagados azules" (blue bloater), caracterizados por 
presentar hipoxemia y, eventualmente, retención de anhídrido carbónico, que habitualmente se complican con hipertensión pulmonar y falla cardiaca derecha. Son pacientes tosedores y expectoradores permanentes y B) "Sopladores rosados" (pink puffer) caracterizados por alta frecuencia de caquexia, gases arteriales relativamente conservados, con importante disnea y habitualmente con enfisema pulmonar ${ }^{26}$.

Estos dos fenotipos pueden representar diferentes manifestaciones sistémicas de la EPOC, pero también muchos pacientes con manifestaciones sistémicas, no tienen las características de estos morfotipos.

Está en curso el estudio ECLIPSE, que permitirá conocer con mayor detalle los distintos fenotipos de esta enfermedad, entregando información de biomarcadores y parámetros que indiquen progresión de la enfermedad e información genética, lo que significará conocer mejor la heterogeneidad de la EPOC $^{27}$. Un reporte inicial de este estudio ${ }^{28}$ muestra que los pacientes con exacerbaciones severas de EPOC tienen enfermedad más avanzada, mayor alteración de calidad de vida y sufren más depresión. Otro reporte de esta cohorte ${ }^{29}$ muestra que pacientes que caminan menos de 350 metros en el test de marcha de 6 minutos tienen más enfisema en la tomografía computada de tórax, más depresión y mayor disnea.

La iniciativa $\mathrm{GOLD}^{6}$ caracteriza a los pacientes EPOC por la limitación al flujo aéreo, específicamente por el $\mathrm{VEF}_{1}$. Se ha sugerido que este parámetro no muestra una vista panorámica de la complejidad del EPOC. Aun más, un editorial de New England Journal of Medicine ${ }^{30}$ ha manifestado que "el VEF no captura ni comunica la heterogeneidad de la EPOC", afirmación refrendada por la cohorte ECLIPSE ${ }^{7}$. Por lo anterior se ha descrito un sistema simple de clasificación multidimensional, llamado BODE, que incluye el índice de masa corporal, el grado de obstrucción dado por el $\mathrm{VEF}_{1}$, el grado de disnea y los metros caminados en el test de marcha de 6 minutos (Figura 3). Este índice es mejor que el $\mathrm{VEF}_{1}$ en predecir el riesgo de muerte en pacientes con EPOC y ha permitido caracterizar mejor a pacientes en estadios GOLD III y IV, respecto su evolución clínica ${ }^{31}$.

También se ha demostrado que no hay correlación entre el nivel de $\mathrm{VEF}_{1}$ y la calidad de vida relacionada con la salud. Hay varios instrumentos para medir esta variable. El más utilizado es el cuestionario de Saint George (SGRQ), instrumento auto-administrado y supervisado que contiene 3 dominios: síntomas, actividad e impacto ${ }^{32}$ y es de gran utilidad para medir la repercusión clínica de las terapias sobre la calidad de vida.

\section{Comorbilidades y consecuencias sistémicas}

Una proporción importante de los pacientes con EPOC presentan alteraciones extra-pulmonares. En EPOC avanzada es común la pérdida de peso y la caquexia. También se observa, en mayor proporción respecto población de edad similar, una disminución de la masa muscular y mayor frecuencia de comorbilidades como osteoporosis, insuficiencia cardiaca, enfermedad cardiaca isquémica, accidente vascular cerebral, depresión y cáncer ${ }^{33}$. Un estudio español ${ }^{34}$, mostró en pacientes con EPOC exacerbado una alta frecuencia de hipertensión arterial, de insuficiencia cardiaca y de diabetes mellitus. Otro estudio ${ }^{35}$ encontró que la causa de muerte en pacientes EPOC exacerbados era insuficiencia cardiaca en $37 \%$ y que sólo 14\% fallecía por insuficiencia respiratoria secundaria a EPOC.

La teoría más aceptada respecto a la respuesta inflamatoria pulmonar anómala, es la llamada "amplificación de la respuesta fisiológica" que se observa frente a estímulos inflamatorios, por ejemplo, el humo del cigarrillo. Por razones aún

\begin{tabular}{|lcccc|}
\hline Puntos variables Índice BODE & $\mathbf{0}$ & $\mathbf{1}$ & $\mathbf{2}$ & $\mathbf{3}$ \\
VEF $_{1}$ (\% del predicho) & $\geq 65$ & $50-64$ & $36-49$ & $\leq 35$ \\
Distancia caminada en $6 \min (\mathrm{m})$ & $\geq 350$ & $250-349$ & $150-249$ & $\leq 149$ \\
Escala disnea MMRC & $0-1$ & 2 & 3 & 4 \\
Índice de masa corporal & $>21$ & $\leq 21$ & & \\
\hline
\end{tabular}

Figura 3. Índice BODE: Variables y puntos de valor usando Índice masa corporal, Metros caminados en 6 minutos, Escala disnea MMRC y Porcentaje del VEF, post-broncodilatador. Adaptado con permiso de referencia 31. 
desconocidas, el equilibrio daño/reparación se torna aberrante, amplificándose y persistiendo a pesar de abandonar el hábito ${ }^{22}$. Esto sugiere que la inflamación crónica que ocurre en la EPOC pudiera estar asociada a un mecanismo de autoperpetuación.

Otra teoría es el fenómeno de "derramamiento" del proceso inflamatorio de la vía aérea, parénquima y circulación pulmonar sobre la circulación sistémica. Mediadores inflamatorios y productos del estrés oxidativo pulmonar son liberados en la circulación sistémica a través de la circulación pulmonar pre-activando a los leucocitos de la sangre periférica ${ }^{36}$. Sin embargo, para otros autores, las manifestaciones pulmonares de la EPOC son una forma más de la expresión de un estado inflamatorio "sistémico", con múltiple compromiso orgánico $^{37}$, concluyendo que la EPOC no es una enfermedad sistémica, pero que tiene importantes consecuencias sistémicas.

\section{Nuevos conocimientos en terapia farmacológica}

En los últimos años ha aparecido mucha investigación farmacológica en EPOC, cada uno con importante número de pacientes y con diferentes objetivos primarios.

Los dos "mega-protocolos" que han dado lugar a un importante número de análisis y publicaciones posteriores, son TORCH (Towards a Revolution in COPD Health) y UPLIFT (Understanding Potential Long-Term Impacts on Function with Tiotropium).

TORCH $^{11}$ es un protocolo de 3 años, aleatorio doble ciego, que comparó fluticasona más salmeterol contra placebo o fluticasona o salmeterol, por separado, todos inhalados, y su objetivo primario era mortalidad. No se demostró impacto sobre la mortalidad, pero hubo disminución del número de exacerbaciones anuales en el grupo tratado con fluticasona/salmeterol. Sin embargo, se observó un aumento de neumonías en los grupos tratados con fluticasona y fluticasona/salmeterol.

UPLIFT $^{38}$ es un protocolo de 4 años, aleatorio doble ciego, que comparó tiotropio inhalado o placebo, contra la terapia habitual (menos anticolinérgicos inhalados) y su objetivo primario era impactar sobre la velocidad de declinación anual del $V_{E F}$. Se demostró que la terapia con tiotropio se asocia a mejora en la función pulmonar, en calidad de vida y presentan menos exacerbaciones, pero no se encontró reducción en la velocidad de declinación del $\mathrm{VEF}_{1}$. No se encontraron efectos cardiovasculares asociados ni mayor incidencia de neumonías. En un análisis posterior ${ }^{39}$ se encontró que los pacientes tratados con tiotropio tenían un "hazard ratio" de mortalidad de causa respiratoria y cardiaca de 0,86 comparado con la terapia habitual. Los autores concluyen que tiotropio se asocia con una disminución de la mortalidad en pacientes con EPOC.

Decramer publicó recientemente un artículo ${ }^{40}$ que analiza un sub-grupo del protocolo UPLIFT: pacientes con EPOC moderado ( $\mathrm{VEF}_{1}$ postbroncodilatador entre 50 y $70 \%$ ), encontrando que tiotropio reduce el número de exacerbaciones, mejora el estado de salud y se asocia con una pequeña disminución de la mortalidad por todas las causas, comparado con placebo. Por la baja incidencia de mortalidad, la diferencia no resultó estadísticamente significativa. Esto es relevante, ya que ha puesto en discusión el beneficio del inicio temprano de la terapia farmacológica en pacientes con EPOC, como se analiza en una reciente editorial ${ }^{41}$.

También está el estudio OPTIMAL ${ }^{42}$, con un año de seguimiento, que comparó el uso de fluticasona/salmeterol más tiotropio (llamada "triterapia") con tiotropio en monoterapia, todos inhalados. Se demostró que triterapia no disminuye el número de exacerbaciones anuales pero mejora la función pulmonar, la calidad de vida y la tasa de hospitalización en EPOC moderados y severos.

Finalmente, aparece el estudio INSPIRE ${ }^{43}$ que compara tiotropio y fluticasona/salmeterol respecto la disminución de exacerbaciones EPOC, no encontrando diferencias significativas entre ambas terapias, en la variable señalada.

\section{Rehabilitación pulmonar}

La rehabilitación pulmonar tiene beneficios probados en el manejo de los pacientes portadores de EPOC. Esta es la intervención que posiblemente tiene más impacto en la calidad de vida, junto con el abandono del hábito tabáquico y la promoción del buen uso de los fármacos. Tiene impacto en la desensibilización central de la disnea, disminuye 
la ansiedad y la depresión, reduce la hiperinflación dinámica y mejora la función del músculo esquelético ${ }^{44}$. En las guías $\mathrm{GOLD}^{6}$ se recomienda agregar desde el estadio II y Celli ${ }^{45}$, en su algoritmo de manejo (Figura 4), recomienda considerar la rehabilitación pulmonar cuando se detecta BODE 3 en un paciente con EPOC.

\section{Terapias farmacológicas no respiratorias}

Está demostrado que el uso de beta-bloqueadores cardio-selectivos reduce el riesgo de muerte por hipertensión arterial, insuficiencia cardiaca y enfermedad coronaria en pacientes con $\mathrm{EPOC}^{46}$.
El uso de estos medicamentos no provoca efectos adversos sobre la función pulmonar o sobre los síntomas respiratorios comparados con placebo en pacientes con EPOC, sin embargo, deben ser usados en dosis bajas y titulación progresiva, vigilando función pulmonar y síntomas respiratorios ${ }^{47}$.

Se ha demostrado que el uso de estatinas en pacientes con EPOC se asocia a mejor sobrevida después de una exacerbación, independiente que el paciente sea portador de enfermedad isquémica cardiaca ${ }^{48}$. Sin embargo, todos estos efectos pleiomórficos de las estatinas requerirán estudios prospectivos controlados, especialmente para ver los efectos sobre las complicaciones sistémicas y las comorbilidades ${ }^{36}$.

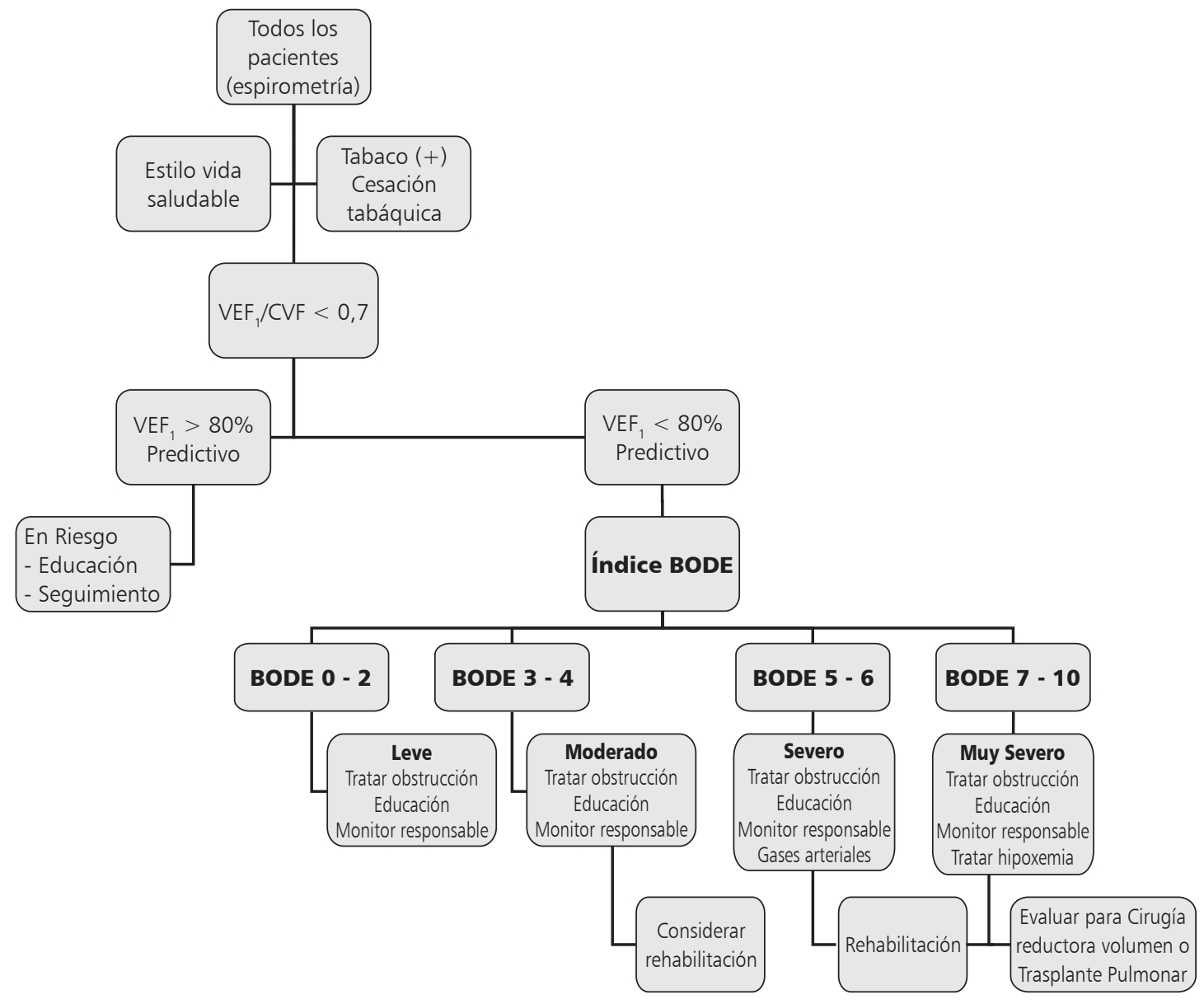

Figura 4. Algoritmo esquemático para el manejo de pacientes con EPOC. Adaptado con permiso de referencia 45. 


\section{Oxigenoterapia}

Una revisión basada en los resultados de 6 estudios clínicos aleatorios y controlados mostró beneficios de la oxigenoterapia domiciliaria (OD) sobre la mortalidad, en un grupo seleccionado de pacientes con EPOC e hipoxemia severa, es decir, presión arterial de oxígeno $\left(\mathrm{PaO}_{2}\right)$, menor de 55 $\mathrm{mmHg}^{49}$. La OD debe ser prescrita por lo menos 15 horas al día, aunque algunos autores consideran que el uso de 24 horas pudiera ser más beneficioso. OD no parece mejorar la sobrevida en pacientes con hipoxemia leve o moderada o en aquellos que presentan desaturación arterial de oxígeno sólo nocturna. OD también redujo el número de hospitalizaciones, mejoró la capacidad de ejercicio y la calidad relacionada con la salud ${ }^{50}$.

\section{El futuro}

El desafío es encontrar una terapia farmacológica que impacte en la mortalidad específica por EPOC. Posiblemente el camino es encontrar antagonistas efectivos de los mediadores y de las proteasas o drogas antiinflamatorias ${ }^{51}$. Se ha investigado antioxidantes, inhibidores de fosfodiesterasa 4, de leucotrienos B4, de factor necrosis tumoral-alfa y de metaloproteinasas, entre otros, encontrando efectos sobre la declinación del $\mathrm{VEF}_{1}$, menos exacerbaciones y mejoría en calidad de vida, pero sin impacto en la mortalidad por EPOC $^{52}$.

Finalmente, quedan algunas preguntas: ¿Por qué sólo algunos fumadores desarrollan EPOC clínicamente significativo?, ¿Por qué hay gran heterogeneidad en la presentación de EPOC?, ¿Cuáles vías patogénicas son críticas y como pueden ser moduladas terapéuticamente?, ¿Por qué la enfermedad continúa progresando después de la cesación tabáquica?, ¿Cómo el daño pulmonar que caracteriza la EPOC puede ser revertido? Estudios futuros nos darán estas respuestas.

Por otro lado, hoy sabemos que los nuevos tratamientos sólo afectan una parte de esta compleja enfermedad. La comprensión, en el futuro, de las variantes fenotípicas y las asociaciones genéticas permitirán el diseño de grandes ensayos clínicos centrados en estos puntos. Además, como los polimorfismos genéticos pueden dictar la respuesta al tratamiento, es evidente que la caracterización genotípica de estos pacientes será clave para manejar la EPOC en el futuro.

\section{Conclusión}

La EPOC es una enfermedad multidimensional con un importante componente inflamatorio. Hoy tenemos avances importantes en terapia farmacológica con los mega-protocolos publicados. Tenemos nuevas herramientas terapéuticas no farmacológicas que tienen un importante impacto en estos pacientes y claramente la rehabilitación debe estar dentro de los programas terapéuticos de EPOC. El gran desafío futuro es tener terapias que impacten sobre la mortalidad por EPOC. Por tanto, no hay razón para tener una actitud nihilista al enfrentar a estos pacientes, claramente hay que ser optimista.

Hay evidencia para ello en este nuevo siglo.

\section{Referencias}

1. Murray C, López A. Alternative projections of mortality and disability by cause 1990-2020: Global Burden of Disease Study. Lancet 1997; 349: 1498-504.

2. Halbert R, Natoli J, Gano A, Badamgarav E, Buist S, Mannino D. Global burden of COPD: systematic review and meta-analysis. Eur Respir J 2006; 28: 523-32.

3. Buist S, Mc Burnie M, Vollmer W, Gillespie SS, Burney $\mathrm{P}$, Mannino $\mathrm{D}$, et al. International variation in the prevalence of COPD (The BOLD Study): a population-based prevalence study. Lancet 2007; 370: 741-50.

4. Meneses A, Pérez-Padilla R, Jardim J, Muiño A, López M, Valdivia G, et al. Chronic obstructive pulmonary disease in five Latin American cities (the PLATINO study): a prevalence study. Lancet 2005; 366: 1875-81.

5. Jemal A, Ward E, Yongping H, Thun M. Trends in the Leading Causes of Death in the United States, 19702002. JAMA 2005; 294: 1255-9.

6. Rabe K, Hurd S, Anzueto A, Barnes P, Buist S, Calverley $\mathrm{P}$, et al. Global Strategy for the Diagnosis, Management, and Prevention of Chronic Obstructive Pulmonary Disease. Am J Respir Crit Care Med 2007; 176: 532-55.

7. Agusti A, Calverley P, Celli B, Coxson H, Edwards L, Lomas D, et al. Characterisation of COPD heterogeneity in the ECLIPSE cohorte. Respiratory Research 2010; 11: 122.

8. Tashkin D, Celli B, Decramer M, Liu D, Burkhart D, Cassino $\mathrm{C}$, et al. Bronchodilator responsiveness in patients with COPD. Eur Respir J 2008; 31: 742-50. 
9. Vestbo J. The TORCH (towards a revolution in COPD health) survival study protocol. Eur Respir J 2004; 24: 206-10.

10. Cazzola M, Mac Nee W, Martínez F, Rabe K, Franciosi L, Barnes P, et al. Outcomes for COPD pharmacological trials: from lung function to biomarkers Eur Respir J 2008; 31: 416-69.

11. Calverley P, Anderson J, Celli B, Ferguson G, Jenkins C, Jones P. Salmeterol and Fluticasone Propionate and Survival in Chronic Obstructive Pulmonary Disease. N Engl J Med 2007; 356: 775-89.

12. Menezes A, Muino A, López M, Pérez-Padilla R, Montes de Oca M, Talamo C. A Prospective study on COPD and risk of mortality five years later, disponible en: http:// www.platinoalat.org/docs/poster_ERS_Viena_mortality_2009.ppt. Consultado 23 diciembre 2009.

13. Fletcher C, Peto R. The Natural History of Chronis Bronquitis and Enphysema. BMJ 1977; 1: 1645-8.

14. Petty T. COPD in Perspective. Chest 2002; 121: 116S-20S.

15. Rodríguez-Roisin R, Drakulovic M, Rodríguez D, Roca J, Barberà J, Wagner P. Ventilation- perfusion imbalance and chonic obstructive pulmonary disease staging severity. J Appl Phisiol 2009; 106: 1902-8.

16. Walters J, Hansen E, Walters H, Wood-Baker R. Underdiagnosis of chronic obstructive pulmonary disease: A qualitative study in primary care. Respiratory Medicine 2008; 102: 738-43.

17. Tálamo C, Montes de Oca M, Halbert R, Pérez-Padilla R, Jardim J, Muiño A, et al. Diagnostic Labeling of COPD in Five Latin American Cities. Chest 2007; 131: 60-7.

18. Montes de Oca M, Talamo C, Halbert R, Pérez-Padilla R, López M, Muiño A, et al. Health status perception and airflow obstruction in five Latin American cities: The PLATINO study. Respiratory Medicine 2009; 103: 1376-82.

19. Celli B. Chronic Obstructive Pulmonary Disease: From Unjustified Nihilism to Evidence-based Optimism; Proc Am Thorac Soc 2006; 3: 58-65.

20. Hallberg J, Dominicus A, Eriksson U, Gerhardsson de Verdier M, Pedersen N, Dahlbäck M, et al. Interaction between Smoking and Genetic Factors in the Development of Chronic Bronchitis. Am J Respir Crit Care Med 2008; 177: 486-90.

21. Barnes P. Chronic Obstructive Pulmonary Disease. N Engl J Med 2000; 343: 269-80.

22. Hogg J, Chu F, Utokaparch S, Woods R, Elliott M, Buzatu $\mathrm{L}$, et al. The nature of small-airway obstruction in chronic obstructive pulmonary disease. N Engl J Med 2004; 350: 2645-53.

23. Brusselle G, Demoor T, Bracke K, Brandsma C, Timens W. Lymphoid follicles in (very) severe COPD: beneficial or harmful?. Eur Respir J 2009; 34: 219-30.

24. Feghali-Bostwick C, Gadgil A, Otterbein L, Pilewski J, Stoner M, Csizmadia E, et al. Autoantibodies in Patients with Chronic Obstructive Pulmonary Disease. Am J Respir Crit Care Med 2008; 177: 156-63.

25. Cosio M, Saetta M, Agustí A. Immunologic Aspects of Chronic Obstructive Pulmonary Disease. N Engl J Med 2009; 360: 2445-54.

26. Rennard S. Looking at the Patient-Approaching the Problem of COPD. N Engl J Med 2004; 350: 965-6.

27. Vestbo J, Anderson W, Coxson H, Crim C, Dawber F, Edwards L, et al. Evaluation of COPD Longitudinally to Identify Predictive Surrogate End-points (ECLIPSE). Eur Respir J 2008; 31: 869-73.

28. Anzueto A, Müllerova H, Locantore N, Hurst J, Wedzicha J, Vestbo J. Occurrence And Descriptors Of Severe COPD Exacerbations In The ECLIPSE Cohort. Am J Respir Crit Care Med 181; 2010: A2388.

29. Spruit M, Watkins M, Edwards L, Vestbo J, Calverley P, Pinto-Plata V, et al. Determinants of poor 6-min walking distance in patients with COPD: The ECLIPSE cohort. Respiratory Medicine 2010; 104: 849-57.

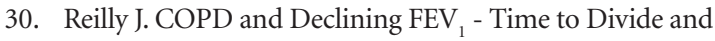
Conquer? N Engl J Med 2008; 359: 1616-8.

31. Celli B, Cote C, Marín J, Casanova C, Montes de Oca M, Méndez R, et al. The body-mass index, airflow obstruction, dyspnea, and exercise capacity index in chronic obstructive pulmonary disease. N Engl J Med 2004; 350: 1005-12.

32. Jones P, Quirk F, Baveystock C. A self-complete measure of health status for chronic airflow limitation. The St George's Respiratory Questionnaire. Am Rev Respir Dis 1992; 145: 1321-7.

33. Tkác J, Man P, Sin D. Systemic consequences of COPD Therapeutic Advances in Respiratory Disease 2007; 1: 47-59.

34. Almagro P, López García F, Cabrera F, Montero L, Morchón D, Diez J, et al. Comorbidity and gender-related differences in patients hospitalized for COPD. The ECCO study. Respiratory Medicine 2010; 104: 253-9.

35. Zvezdin B, Milutinov S, Kojicic M, Hadnadejev M, Hromis S, Markovic M, et al. Early Death in Patients Hospitalized With A Postmortem Analysis of Major Causes of COPD Exacerbation. Chest 2009; 136: 376-80.

36. Barnes P, Celli B. Systemic manifestations and comorbidities of COPD. Eur Respir J 2009; 33: 1165-85.

37. Sevenoaks M, Stockley R. Chronic obstructive pulmonary disease, inflammation and co-morbidity -a common inflammatory phenotype? Respir Res 2006; 7: 70.

38. Tashkin D, Celli B, Senn S, Burkhart D, Kesten S, Menjoge S, et al. A 4-Year Trial of Tiotropium in Chronic 
Enfermedad pulmonar obstructiva crónica - R. Silva

Obstructive Pulmonary Disease N Engl J Med 2008; 359: 1543-54.

39. Celli B, Decramer M, Kesten S, Liu D, Mehra S, Taskin D. Mortality in the 4-Year Trial of Tiotropium (UPLIFT) in Patients with Chronic Obstructive Pulmonary Disease Am J Respir Crit Care Med 2009; 180: 948-55.

40. Decramer M, Celli B, Kesten S, Lystig T, Mehra S, Tashkin D. Effect of tiotropium on outcomes in patients with moderate chronic obstructive pulmonary disease (UPLIFT): a prespecified subgroup analysis of a randomised controlled trial. Lancet 2009; 374: 1171-8.

41. Davies L, Calverley P. UPLIFTing care for chronic obstructive pulmonary disease; Lancet 2009; 374: 1129-30.

42. Aaron A, Vandemheen K, Fergusso D, Maltais F, Bourbea J, Goldstein R, et al. Tiotropium in Combination with Placebo, Salmeterol, or Fluticasone-Salmeterol for Treatment of Chronic Obstructive Pulmonary Disease: A Randomized Trial Ann Intern Med 2007; 146: 545-55.

43. Wedzicha J, Calverley P, Seemungal T, Hagan G, Ansari Z, Stockley R. The Prevention of Chronic Obstructive Pulmonary Disease Exacerbations by Salmeterol/Fluticasone Propionate or Tiotropium Bromide. Am J Respir Crit Care Med 2008; 177: 19-26.

44. Casaburi R, Zu-Wallack R. Pulmonary Rehabilitation for Management of Chronic Obstructive Pulmonary
Disease. N Engl J Med 2009; 360: 1329-35.

45. Celli B. Update on the Management of COPD. Chest 2008; 133; 1451-62.

46. Salpeter S, Ormiston T, Salpeter E. Cardioselective betablockers for chronic obstructive pulmonary disease (Review). Cochrane Database of Systematic Reviews 2005, Issue 4.

47. Cazzola M. B-Blockers Are Safe in Patients with Chronic Obstructive Pulmonary Disease, But Only with Caution. Am J Respir Crit Care Med 2008; 178: 661-6.

48. Soyseth V, Brekke P, Smith P, Omland T. Statin use is associated with reduced mortality in COPD. Eur Respir J 2007; 29: 279-83.

49. Cranston J, Crockett A, Moss J, Alpers J. Domiciliary oxygen for chronic obstructive pulmonary disease. Cochrane Database Syst Rev 2005; 4: CD001744.

50. Haidl P, Clement C, Wiese C, Dellweg D, Kohler D. Long-term oxygen therapy stops the natural decline of endurance in COPD patients with reversible hypercapnia. Respiration 2004; 71: 342-7.

51. Barnes P. Chronic Obstructive Pulmonary Disease. N Engl J Med 2000; 343: 269-80.

52. Woods A, Stockley R. Unifying the genetics, co-morbidities and management of COPD; Therapeutic Advances in Respiratory Disease 2008; 2: 113-7. 